\section{The Importance of Linking Primary and Secondary Electronic Medical Records}

\section{To the Editor:}

We would like to congratulate C. John Michet $3 \mathrm{rd}$, et al ${ }^{1}$ on their excellent paper highlighting the issues of relying on hospital data for epidemiological studies. Routinely collected medical data, such as those found in hospital case notes, are an increasingly important resource for research, and therefore understanding the strengths and weaknesses of these data is important to clinicians and researchers alike.

Hospital records are particularly important and useful as data sources when studying conditions predominately diagnosed and managed in specialist care settings, such as rheumatoid arthritis and giant cell arteritis. However, for some common rheumatological conditions such as gout and polymyalgia rheumatica, the majority of patient encounters occur in primary care settings because many such patients will never be referred to specialist care; thus these cases will not be identified by hospital records. In these conditions, research solely using records from specialist care settings will be misleading, and likely include only patients with diagnostic uncertainty, poor or atypical treatment response, or more severe disease.

In the United Kingdom, we are fortunate to have excellent primary care medical records that are available for research purposes. As concluded by the authors, we would also advocate judicious use of hospital data. We would like to encourage more frequent use of data from primary care, be it alone or in combination with hospital data, because those data are most appropriate for the research question. The time has come for all researchers, reviewers, and readers to be fully aware of and acknowledge the strengths and limitations of the multitude of data sources available to us, and to use the sources most appropriate to our questions, not just those that are most convenient.

SARA MULLER, PhD, Arthritis Research UK Primary Care Centre, Research Institute for Primary Care and Health Sciences, Keele University, Keele, UK; SAMANTHA L. HIDER, MBBS, PhD, FRCP, Arthritis Research UK Primary Care Centre, Research Institute for Primary Care and Health Sciences, Keele University, Keele, UK; CHRISTIAN D. MALLEN, MBBS, PhD, FRCGP, Arthritis Research UK Primary Care Centre, Research Institute for Primary Care and Health Sciences, Keele University, Keele, UK. CDM is funded by a UK National Institute for Health Research (NIHR) Research Professorship (NIHR-RP-2014-04-026), the NIHR Collaborations for Leadership in Applied Health Research and Care West Midlands, and the NIHR School for Primary Care Research. Address correspondence to Professor C.D. Mallen, Research Institute for Primary Care and Health Sciences, Keele University, Keele, Staffordshire ST55 BG, UK. E-mail: c.d.mallen@keele.ac.uk

\section{REFERENCE}

1. Michet CJ 3rd, Crowson CS, Achenbach SJ, Matteson EL. The detection of rheumatic disease through hospital diagnoses with examples of rheumatoid arthritis and giant cell arteritis: what are we missing? J Rheumatol 2015;42:2071-4

J Rheumatol 2016;43:6; doi:10.3899/jrheum.151336 